\title{
ADMISSIBLE CONDITIONS FOR PARABOLIC EQUATIONS DEGENERATING AT INFINITY
}

\author{
SH. KAMIN, M. A. POZIO, AND A. TESEI
}

\begin{abstract}
Well-posedness in $L^{\infty}\left(\mathbb{R}^{n}\right)(n \geq 3)$ of the Cauchy problem is studied for a class of linear parabolic equations with variable density. In view of degeneracy at infinity, some conditions at infinity are possibly needed to make the problem well-posed. Existence and uniqueness results are proved for bounded solutions that satisfy either Dirichlet or Neumann conditions at infinity.
\end{abstract}

\section{$\S 1$. INTRODUCTION}

We investigate existence and uniqueness for bounded solutions of the parabolic Cauchy problem

$$
\begin{cases}\rho \partial_{t} u=\Delta u & \text { in } \mathbb{R}^{n} \times \mathbb{R}_{+}=: S, \\ u=u_{0} & \text { in } \mathbb{R}^{n} \times\{0\} \quad(n \geq 3) .\end{cases}
$$

Concerning the coefficient $\rho=\rho(x)$ and the initial data $u_{0}$ in (1.1), we always assume the following:

$$
\left\{\begin{array}{l}
\text { (i) } \quad \rho \in C_{\text {loc }}^{1+\alpha}\left(\mathbb{R}^{n}\right), \quad \rho>0 \quad \text { in } \mathbb{R}^{n} ; \\
\text { (ii) } u_{0} \in C_{\text {loc }}^{\alpha}\left(\mathbb{R}^{n}\right) \cap L^{\infty}\left(\mathbb{R}^{n}\right) \quad(\alpha \in(0,1)) .
\end{array}\right.
$$

The solutions of problem (1.1) are always meant in the classical sense.

As is well known, problem (1.1) can be ill posed in the set of bounded solutions, depending on the behavior of $\rho$ as $r:=|x| \rightarrow \infty$ and on the space dimension $n$. To be specific, it is well posed in the above class for any smooth, positive $\rho$ if $n \leq 2$ whereas, if $n \geq 3$ and $\rho$ tends to 0 sufficiently fast as $r \rightarrow \infty$ (depending on $n$ ), some conditions at infinity are needed to restore well-posedness (see [3]-[11], [14]-[15], [17] and the references therein). In the papers mentioned above, such conditions are of Dirichlet type and homogeneous, for they require the unknown to vanish at infinity in a suitable sense.

However, it is quite natural to consider conditions of a different type. In this paper we address the uniqueness of bounded solutions of (1.1) that satisfy at infinity either inhomogeneous conditions of Dirichlet type (see Subsection 1.1), or conditions of Neumann type (see Subsection 1.2). Remarkably, the results obtained for the former enable us to address the latter (see the proof of Theorem 1.5). It is also worthy of mention that the solutions satisfying conditions of Dirichlet type are uniquely determined by the limiting value at infinity of their spherical means (see Theorems 1.1, 1.3).

In the present paper we only deal with the model equation in (1.1); however, similar results should be valid for a wider class of quasilinear parabolic equations.

2000 Mathematics Subject Classification. Primary 35K15, 35K65.

Key words and phrases. Parabolic Cauchy problem, linear parabolic equations with variable density, bounded solutions.

Partially supported by RTN Contract HPRN-CT-2002-00274. 
1.1. Dirichlet conditions. Besides condition $\left(\mathrm{H}_{0}\right)$, to deal with this case we assume that

$$
\Gamma * \rho \in L^{\infty}\left(\mathbb{R}^{n}\right),
$$

where $\Gamma$ denotes the fundamental solution of the Laplace equation in $\mathbb{R}^{n}$. As was observed in [1], condition $\left(\mathrm{H}_{1}\right)$ is satisfied if and only if the equation

$$
-\Delta v=\rho \quad \text { in } \mathbb{R}^{n}
$$

has a bounded solution.

Set $B_{R}:=\left\{x \in \mathbb{R}^{n}|| x \mid<R\right\}, R>0$. We denote by

$$
f_{\partial B_{R}} v d \sigma:=\frac{1}{\left|\partial B_{R}\right|} \int_{\partial B_{R}} v d \sigma
$$

the mean of a function $v$ on the sphere $\partial B_{R}$.

Our first result shows that any bounded solution of problem (1.1) has a trace at infinity in a suitable sense.

Theorem 1.1. Let assumptions $\left(\mathrm{H}_{0}\right)$ and $\left(\mathrm{H}_{1}\right)$ be satisfied, and let $u$ be any bounded solution of problem (1.1). Set

$$
U(x, t):=\int_{0}^{t} u(x, \tau) d \tau \quad((x, t) \in S) .
$$

Then there exists $A \in \operatorname{Lip}\left(\overline{\mathbb{R}}_{+}\right)$with $A(0)=0$ such that

$$
\lim _{R \rightarrow \infty} f_{\partial B_{R}} U(x, t) d \sigma=A(t) .
$$

Moreover, we have

$$
\lim _{R \rightarrow \infty} f_{\partial B_{R}}|U(x, t)-A(t)| d \sigma=0
$$

uniformly with respect to $t \in[0, T]$ for any $T>0$.

Conversely, it is natural to regard (1.5) as a (possibly inhomogeneous) Dirichlet condition at infinity for any given $A \in \operatorname{Lip}\left(\overline{\mathbb{R}}_{+}\right)$. The existence of a bounded solution of problem (1.1) satisfying such a condition is established in the following theorem.

Theorem 1.2. Let assumptions $\left(\mathrm{H}_{0}\right),\left(\mathrm{H}_{1}\right)$ be satisfied, and let $A \in \operatorname{Lip}\left(\overline{\mathbb{R}}_{+}\right)$with $A(0)=$ 0 . Then there exists a bounded solution u of problem (1.1) satisfying (1.5), with $U$ defined by (1.3).

Imposing condition (1.5) also implies uniqueness, as the following theorem shows.

Theorem 1.3. Let assumptions $\left(\mathrm{H}_{0}\right),\left(\mathrm{H}_{1}\right)$ be satisfied, and let $A \in \operatorname{Lip}\left(\overline{\mathbb{R}}_{+}\right)$with $A(0)=$ 0 . Then there exists at most one bounded solution of (1.1) satisfying (1.5).

We note that a similar uniqueness result concerning positive solutions of the quasilinear equation

$$
\rho \partial_{t} u=\Delta G(u) \text { in } S
$$

(here $G: \mathbb{R}_{+} \rightarrow \mathbb{R}_{+}$is a smooth function satisfying suitable assumptions) was proved in [5] in the particular case where $A \equiv 0$. 
1.2. Neumann conditions. When dealing with conditions of Neumann type, besides $\left(\mathrm{H}_{0}\right)$ we assume that

$$
\rho \in L^{1}\left(\mathbb{R}^{n}\right) \cap L^{\infty}\left(\mathbb{R}^{n}\right)
$$

Observe that condition $\left(\mathrm{H}_{2}\right)$ is stronger than $\left(\mathrm{H}_{1}\right)$. In fact, for any $x \in \mathbb{R}^{n}$ we have

$$
\begin{aligned}
\{\Gamma * \rho\}(x) & =C \int_{|x-y|>1} \frac{\rho(y)}{|x-y|^{n-2}} d y+C \int_{|x-y| \leq 1} \frac{\rho(y)}{|x-y|^{n-2}} d y \\
& \leq C \int_{|x-y|>1} \rho(y) d y+C \int_{|x-y| \leq 1} \frac{\rho(y)}{|x-y|^{n-2}} d y \\
& \leq C\left\{\|\rho\|_{1}+\|\rho\|_{\infty} \frac{\left|\partial B_{1}\right|}{2}\right\}, \quad C>0,
\end{aligned}
$$

and the claim follows.

If $u$ is a bounded solution of problem (1.1), then

$$
\int_{B_{R}} \rho u(x, t) d x-\int_{B_{R}} \rho u_{0} d x=\int_{0}^{t} \int_{\partial B_{R}} \partial_{\nu} u d \sigma d \tau \quad \text { for any } R>0, t>0
$$

here $\partial_{\nu}$ denotes the outer normal derivative at any point of the sphere $\partial B_{R}$. By $\left(\mathrm{H}_{2}\right)$, we have

$$
\lim _{R \rightarrow \infty}\left\{\int_{B_{R}} \rho u(x, t) d x-\int_{B_{R}} \rho u_{0} d x\right\}=\int_{\mathbb{R}^{n}} \rho u(x, t) d x-\int_{\mathbb{R}^{n}} \rho u_{0} d x .
$$

Hence the conservation law

$$
\int_{\mathbb{R}^{n}} \rho u(x, t) d x=\int_{\mathbb{R}^{n}} \rho u_{0} d x \quad(t>0)
$$

is fulfilled if and only if the Neumann condition at infinity

$$
\lim _{R \rightarrow \infty} \int_{0}^{t} \int_{\partial B_{R}} \partial_{\nu} u d \sigma d \tau=0 \quad(t>0)
$$

is satisfied. This motivates the following definition (where, obviously, the letter $\mathcal{N}$ stands for "Neumann").

Definition 1.1. A solution of problem (1.1) belongs to the class $\mathcal{N}$ if it satisfies the conservation law (1.6) for any $t>0$.

The existence of solutions in the class $\mathcal{N}$ is the content of the following theorem, where by $L_{\rho}^{2}\left(\mathbb{R}^{n}\right)$ we denote the weighted Lebesgue space with the norm

$$
\|h\|_{L_{\rho}^{2}}:=\left(\int_{\mathbb{R}^{n}}|h|^{2} \rho d x\right)^{\frac{1}{2}}
$$

Theorem 1.4. Let assumptions $\left(\mathrm{H}_{0}\right)$ and $\left(\mathrm{H}_{2}\right)$ be satisfied. Then there exists a bounded solution $\hat{u} \in \mathcal{N}$ of problem (1.1); moreover, $\hat{u} \in L^{\infty}\left(\mathbb{R}_{+} ; L_{\rho}^{2}\left(\mathbb{R}^{n}\right)\right)$ and $|\nabla \hat{u}| \in L^{2}(S)$.

We shall also prove a uniqueness result.

Theorem 1.5. Let assumptions $\left(\mathrm{H}_{0}\right)$ and $\left(\mathrm{H}_{2}\right)$ be satisfied. Then in the class $\mathcal{N}$ there exists at most one bounded solution of problem (1.1).

Theorems 1.4 and 1.5 immediately imply the following.

Corollary 1.6. Let assumptions $\left(\mathrm{H}_{0}\right)$ and $\left(\mathrm{H}_{2}\right)$ be satisfied. Then any bounded solution $u \in \mathcal{N}$ of problem (1.1) belongs to $L^{\infty}\left(\mathbb{R}_{+} ; L_{\rho}^{2}\left(\mathbb{R}^{n}\right)\right) ;$ moreover, $|\nabla u| \in L^{2}(S)$. 
In view of the conservation law (1.6), it is natural to expect any bounded solution of class $\mathcal{N}$ to converge to the weighted mean of the initial data as $t \rightarrow \infty$. This is established in the following theorem.

Theorem 1.7. Let assumptions $\left(\mathrm{H}_{0}\right)$ and $\left(\mathrm{H}_{2}\right)$ be satisfied. Let $u$ be any bounded solution of class $\mathcal{N}$ to problem (1.1). Then

$$
\lim _{t \rightarrow \infty} u(\cdot, t)=\frac{\int_{\mathbb{R}^{n}} \rho u_{0} d x}{\int_{\mathbb{R}^{n}} \rho d x}
$$

uniformly on compact subsets of $\mathbb{R}^{n}$.

Note that the asymptotic behavior of solutions of (1.1), in dependence on the behavior of $\rho$ at infinity, was investigated in [2] for $n=1$ (see also [6]-[8]).

\section{$\S 2$. Dirichlet conditions: Proofs}

Lemma 2.1. Suppose $f \in L_{\mathrm{loc}}^{\infty}\left(\mathbb{R}^{n}\right)$ and $\Gamma *|f| \in L^{\infty}\left(\mathbb{R}^{n}\right)$. Let $v$ be a bounded solution of the equation

$$
-\Delta v=f \quad \text { in } \mathbb{R}^{n}
$$

Then there exists $A \in \mathbb{R}$ such that

$$
\lim _{R \rightarrow \infty} f_{\partial B_{R}} v d \sigma=A
$$

Moreover,

$$
\lim _{R \rightarrow \infty} f_{\partial B_{R}}|v-A| d \sigma=0
$$

Proof. We set $f^{ \pm}:=\max \{ \pm f, 0\}$ and

$$
\tilde{v}_{ \pm}:=\Gamma * f^{ \pm} \quad \text { in } \mathbb{R}^{n}
$$

where $\Gamma$ denotes the fundamental solution of the Laplace equation in $\mathbb{R}^{n}$; then

$$
-\Delta \tilde{v}_{ \pm}=f^{ \pm} \quad \text { in } \mathbb{R}^{n}
$$

Moreover, since

$$
0 \leq \tilde{v}_{ \pm} \leq \Gamma *|f| \quad \text { in } \mathbb{R}^{n},
$$

we have $\tilde{v}_{ \pm} \in L^{\infty}\left(\mathbb{R}^{n}\right)$. By [1, Lemma A.4],

$$
\lim _{R \rightarrow \infty} f_{\partial B_{R}} \tilde{v}_{ \pm} d \sigma=0
$$

Define $\tilde{v}:=\tilde{v}_{+}-\tilde{v}_{-}$. Then

$\tilde{v} \in L^{\infty}\left(\mathbb{R}^{n}\right)$, and

$$
-\Delta \tilde{v}=f \quad \text { in } \mathbb{R}^{n}
$$

$$
\lim _{R \rightarrow \infty} f_{\partial B_{R}} \tilde{v} d \sigma=0 .
$$

Being a bounded harmonic function in $\mathbb{R}^{n}$, the difference $v-\tilde{v}$ is a constant; namely,

$$
v=\tilde{v}+A \text { in } \mathbb{R}^{n}
$$

for some $A \in \mathbb{R}$. Hence, by (2.6), we have

$$
\lim _{R \rightarrow \infty} f_{\partial B_{R}} v d \sigma=\lim _{R \rightarrow \infty} f_{\partial B_{R}} \tilde{v} d \sigma+A=A .
$$

Clearly, $|\tilde{v}| \leq \tilde{v}_{+}+\tilde{v}_{-}$; now (2.3) follows immediately from (2.5). This proves the result. 
Proof of Theorem 1.1. Integration of the first equation in (1.1) with respect to time gives

$$
-\Delta U(\cdot, t)=\rho\left[u_{0}-u(\cdot, t)\right] \text { in } \mathbb{R}^{n}
$$

for any fixed $t>0$, where $U$ is defined by (1.3). The assumptions $\left(\mathrm{H}_{0}\right),\left(\mathrm{H}_{1}\right)$ and the boundedness of $u$ imply that for any $t>0$ the right-hand side of (2.7) satisfies the conditions of Lemma 2.1. By this lemma, there exists $A: \overline{\mathbb{R}}_{+} \rightarrow \mathbb{R}$ with $A(0)=0$ such that relations (1.4) and (1.5) are true for any $t>0$; in fact, these relations follow from $(2.2)$ and $(2.3)$, respectively.

It remains to prove the following: (i) the function $A: \overline{\mathbb{R}}_{+} \rightarrow \mathbb{R}$ is Lipschitz continuous, and (ii) the convergence in (1.4), (1.5) is uniform with respect to $t \in[0, T]$ for any finite $T>0$. Since $u$ is bounded, we have

$$
|U(x, t)-U(x, s)| \leq\|u\|_{\infty}|t-s| \quad((x, t),(x, s) \in S),
$$

whence

$$
\left|f_{\partial B_{R}}[U(x, t)-U(x, s)] d \sigma\right| \leq\|u\|_{\infty}|t-s| \quad(s, t \geq 0)
$$

for any $R>0$. By (1.4), since $A(0)=0$, we have

$$
A(t)-A(s)=\lim _{R \rightarrow \infty} f_{\partial B_{R}}[U(x, t)-U(x, s)] d \sigma
$$

for any $s, t \geq 0$, and (i) follows. As for (ii), it suffices to observe that the family of functions

$$
f_{R}: \overline{\mathbb{R}}_{+} \rightarrow \mathbb{R}, \quad f_{R}(t):=f_{\partial B_{R}}|U(x, t)-A(t)| d \sigma \quad(R>0)
$$

is uniformly equicontinuous, by inequality (2.8). This completes the proof.

For any $A \in \operatorname{Lip}\left(\overline{\mathbb{R}}_{+}\right)$, the derivative $a \equiv A^{\prime}$ exists almost everywhere and belongs to $L^{\infty}\left(\mathbb{R}_{+}\right)$. Let $a_{R}$ denote a smooth approximation of $a$; we assume that $\left\|a_{R}\right\|_{\infty} \leq\|a\|_{\infty}$ for any $R>0$ and $a_{R} \rightarrow a$ in $L^{1}(0, T)$ for any $T>0$ as $R \rightarrow \infty$. Also, we set $u_{0, R}:=\zeta_{R} u_{0}+\left(1-\zeta_{R}\right) a_{R}(R>0)$, where $\zeta_{R} \in C_{0}^{\infty}\left(B_{R}\right), 0 \leq \zeta_{R} \leq 1, \zeta_{R}=1$ in $B_{R / 2}$.

Fixing an arbitrary $T>0$, for any $R>0$ we consider the following auxiliary problem:

$$
\begin{cases}\rho \partial_{t} u_{R}=\Delta u_{R} & \text { in } B_{R} \times(0, T)=: Q_{R, T}, \\ u_{R}=a_{R}(\cdot) & \text { in } \partial B_{R} \times(0, T), \\ u_{R}=u_{0, R} & \text { in } B_{R} \times\{0\} .\end{cases}
$$

Now we can prove Theorem 1.2.

Proof of Theorem 1.2. Existence, uniqueness, and comparison results for solutions of problem (2.9) can be proved by standard methods. By comparison results, we have

$$
\left|u_{R}\right| \leq\left\|u_{0}\right\|_{\infty}+\|a\|_{\infty} \quad \text { in } Q_{R, T}
$$

The interior estimates for derivatives (see [12]) show that, in any compact set $K \subseteq$ $\mathbb{R}^{n} \times[0, T]$, the first derivatives of $u_{R}$ are also bounded uniformly with respect to $R>0$. Let $R \rightarrow \infty$. By the standard compactness arguments, there exists a subsequence $\left\{u_{R_{k}}\right\}$ that converges uniformly in $K$ to a bounded solution $u$ of problem (1.1).

Let $U$ be defined as in (1.3). Setting

$$
U_{R}(x, t):=\int_{0}^{t} u_{R}(x, \tau) d \tau \quad\left((x, t) \in Q_{R, T}\right),
$$

we observe that $U_{R_{k}} \rightarrow U$ in $\mathbb{R}^{n} \times[0, T]$ as $k \rightarrow \infty$. The conclusion will follow if we prove that the function $U$ satisfies (1.5) uniformly with respect to $t \in[0, T]$. 
It is easily seen that for any $t \in(0, T)$ the function $U_{R}(\cdot, t)$ satisfies the problem

$$
\begin{cases}-\Delta U_{R}(\cdot, t)=\rho\left[u_{0, R}-u_{R}(\cdot, t)\right] & \text { in } B_{R}, \\ U_{R}(\cdot, t)=A_{R}(t) & \text { in } \partial B_{R},\end{cases}
$$

with

$$
A_{R}(t):=\int_{0}^{t} a_{R}(\tau) d \tau \quad(t>0) .
$$

It follows that

$$
U_{R}(x, t)=\int_{B_{R}} \Gamma_{R}(x-y) \rho(y)\left[u_{0, R}(y)-u_{R}(y, t)\right] d y+A_{R}(t) \quad\left((x, t) \in Q_{R, T}\right),
$$

where $\Gamma_{R}$ denotes the Green function of the Laplace equation in $B_{R}$ with the zero Dirichlet boundary conditions. We shall prove the following statement.

Claim. We have

$$
U(x, t)=\left\{\Gamma *\left[\rho\left(u_{0}-u(\cdot, t)\right)\right]\right\}(x)+A(t) \quad\left((x, t) \in \mathbb{R}^{n} \times(0, T)\right) .
$$

From this claim, the conclusion follows. Indeed, since $\Gamma * \rho \in L^{\infty}\left(\mathbb{R}^{n}\right)$ by $\left(\mathrm{H}_{1}\right)$ and $u_{0}, u \in L^{\infty}\left(\mathbb{R}^{n}\right)$ (see $(2.10)$ ), we have $\Gamma *\left[\rho\left|u_{0}-u(\cdot, t)\right|\right] \in L^{\infty}\left(\mathbb{R}^{n}\right)$. Hence, arguing as in the proof of Lemma 2.1 we see that

$$
\lim _{R \rightarrow \infty} f_{\partial B_{R}} \Gamma *\left[\rho\left(u_{0}-u\right)\right] d \sigma=0
$$

for any $t \in[0, T]$. Combined with Theorem 1.1, this gives us (1.5).

It remains to prove the claim. Suppose $R_{0}>0$ is fixed; for any $R>2 R_{0}$ we rewrite (2.13) as follows:

$$
\begin{aligned}
U_{R}(x, t)= & \int_{B_{R_{0}}} \Gamma_{R}(x-y) \rho(y)\left[u_{0}(y)-u_{R}(y, t)\right] d y \\
& +\int_{B_{R} \backslash B_{R_{0}}} \Gamma_{R}(x-y) \rho(y)\left[u_{0, R}(y)-u_{R}(y, t)\right] d y+A_{R}(t) \\
\left((x, t) \in Q_{R, T}\right) &
\end{aligned}
$$

(observe that $u_{0, R}=u_{0}$ in $B_{R_{0}}$ for $R>2 R_{0}$ ). Similarly, we have

$$
\begin{array}{r}
\left\{\Gamma *\left[\rho\left(u_{0}-u(\cdot, t)\right)\right]\right\}(x)= \\
\int_{B_{R_{0}}} \Gamma(x-y) \rho(y)\left[u_{0}(y)-u(y, t)\right] d y \\
+\int_{\mathbb{R}^{n} \backslash B_{R_{0}}} \Gamma(x-y) \rho(y)\left[u_{0}(y)-u(y, t)\right] d y \\
\quad\left((x, t) \in \mathbb{R}^{n} \times(0, T)\right) .
\end{array}
$$

Let $(x, t) \in \mathbb{R}^{n} \times(0, T)$ and $\varepsilon>0$ be fixed arbitrarily; we can choose $R_{0}>0$ such that

$$
\begin{aligned}
& \left|\int_{\mathbb{R}^{n} \backslash B_{R_{0}}} \Gamma(x-y) \rho(y)\left[u_{0}(y)-u(y, t)\right] d y\right|<\varepsilon, \\
& \left|\int_{B_{R} \backslash B_{R_{0}}} \Gamma_{R}(x-y) \rho(y)\left[u_{0, R}(y)-u_{R}(y, t)\right] d y\right|<\varepsilon \quad \text { for any } R>R_{0} .
\end{aligned}
$$

Indeed, inequality $(2.17)$ is true because $\Gamma *\left[\rho\left(u_{0}-u(\cdot, t)\right)\right] \in L^{\infty}\left(\mathbb{R}^{n}\right)$; on the other hand, since $\Gamma * \rho \in L^{\infty}\left(\mathbb{R}^{n}\right)$ by $\left(\mathrm{H}_{1}\right), 0 \leq \Gamma_{R} \leq \Gamma$ in $B_{R}$, and $u_{0, R}, u_{R}(\cdot, t) \in L^{\infty}\left(\mathbb{R}^{n}\right)$ uniformly for $R>0$, it follows that inequality (2.18) is also true. 
We fix $R_{0}$ such that (2.17) and (2.18) are fulfilled. By the dominated convergence theorem,

$$
\lim _{k \rightarrow \infty} \int_{B_{R_{0}}} \Gamma_{R_{k}}(x-y) \rho(y)\left[u_{0}(y)-u_{R_{k}}(y, t)\right] d y=\int_{B_{R_{0}}} \Gamma(x-y) \rho(y)\left[u_{0}(y)-u(y, t)\right] d y .
$$

Then, by (2.15), (2.16) and the above remarks, we have

$$
\lim _{k \rightarrow \infty} U_{R_{k}}(x, t)=U(x, t)=\left\{\Gamma *\left[\rho\left(u_{0}-u(\cdot, t)\right)\right]\right\}(x)+A(t) \quad\left((x, t) \in \mathbb{R}^{n} \times(0, T)\right) .
$$

This completes the proof of the claim; the result follows.

Proof of Theorem 1.3. Let $u_{1}, u_{2}$ be two bounded solutions of problem (1.1). Set

$$
U_{i}(x, t):=\int_{0}^{t} u_{i}(x, \tau) d \tau \quad((x, t) \in S ; i=1,2) .
$$

We assume that

$$
\lim _{R \rightarrow \infty} f_{\partial B_{R}} U_{1}(x, t) d \sigma=\lim _{R \rightarrow \infty} f_{\partial B_{R}} U_{2}(x, t) d \sigma=A(t)
$$

for any $t>0$. Define $w:=u_{1}-u_{2}$; then $w$ satisfies the problem

$$
\begin{cases}\rho \partial_{t} w=\Delta w & \text { in } S \\ w=0 & \text { in } \mathbb{R}^{n} \times\{0\}\end{cases}
$$

Setting

$$
W(x, t):=\int_{0}^{t} w(x, \tau) d \tau=U_{1}(x, t)-U_{2}(x, t) \quad((x, t) \in S)
$$

and recalling (1.5), we see that

$$
\begin{aligned}
& \lim _{R \rightarrow \infty} f_{\partial B_{R}}|W(x, t)| d \sigma=\lim _{R \rightarrow \infty} f_{\partial B_{R}}\left|U_{1}(x, t)-U_{2}(x, t)\right| d \sigma \\
& \quad \leq \lim _{R \rightarrow \infty}\left\{f_{\partial B_{R}}\left|U_{1}(x, t)-A(t)\right| d \sigma+f_{\partial B_{R}}\left|U_{2}(x, t)-A(t)\right| d \sigma\right\}=0
\end{aligned}
$$

uniformly with respect to $t \in[0, T]$ for any $T>0$.

We prove that $w \equiv 0$. For this, it suffices to check that

$$
\int_{0}^{T} \int_{\mathbb{R}^{n}} w F d x d t=0
$$

for any $T>0$ and any test function $F=F(x, t) \in C_{0}^{\infty}\left(\mathbb{R}^{n} \times(0, T)\right)$. Without loss of generality we may assume that $\operatorname{supp} F \subseteq B_{R_{0}} \times(0, T)$ for some $R_{0}>0$ ( $T>0$ is fixed). Consider the solution $\psi \equiv \psi_{R}$ of the backward problem

$$
\begin{cases}\rho \partial_{t} \psi+\Delta \psi=-F & \text { in } Q_{R, T}, \\ \psi=0 & \text { in } \partial B_{R} \times(0, T), \\ \psi=0 & \text { in } B_{R} \times\{T\},\end{cases}
$$

where $R>R_{0}$ (the index $R$ is omitted in what follows). Then

$$
\int_{0}^{T} \int_{B_{R}} w F d x d t=-\int_{0}^{T} \int_{\partial B_{R}} w \partial_{\nu} \psi d \sigma d t
$$

we have used the fact that $w=0$ in $B_{R} \times\{0\}$ and $\psi=0$ in $\left(B_{R} \times\{T\}\right) \cup\left(\partial B_{R} \times(0, T)\right)$. Identity (2.24) shows that the conclusion will follow if we check the relation

$$
\lim _{R \rightarrow \infty} \int_{0}^{T} \int_{\partial B_{R}} w \partial_{\nu} \psi d \sigma d t=0 .
$$


Integrating by parts over $(0, T)$ yields

$$
\int_{0}^{T} \int_{\partial B_{R}} w \partial_{\nu} \psi d \sigma d t=\int_{\partial B_{R}} \int_{0}^{T} \partial_{t} W \partial_{\nu} \psi d t d \sigma=-\int_{\partial B_{R}} \int_{0}^{T} W \partial_{t}\left(\partial_{\nu} \psi\right) d t d \sigma
$$

we have used the fact that $W=0$ in $\partial B_{R} \times\{0\}$ and $\partial_{\nu} \psi=0$ in $\partial B_{R} \times\{T\}$. The regularity of $\rho$ (see assumption $\left.\left(\mathrm{H}_{0}\right)-(\mathrm{i})\right)$ implies $\psi \in C^{2}\left(\bar{Q}_{R, T}\right)$. Consequently,

$$
\left|\int_{0}^{T} \int_{\partial B_{R}} w \partial_{\nu} \psi d \sigma d t\right| \leq \max _{\partial B_{R} \times[0, T]}\left|\partial_{\nu}\left(\partial_{t} \psi\right)\right| \int_{0}^{T} \int_{\partial B_{R}}|W| d \sigma d t .
$$

Set $\varphi \equiv \varphi_{R}:=\partial_{t} \psi$; from (2.23) we see that $\varphi$ satisfies the backward problem

$$
\begin{cases}\rho \partial_{t} \varphi+\Delta \varphi=-\partial_{t} F & \text { in } Q_{R, T} \\ \varphi=0 & \text { in } \partial B_{R} \times(0, T) \\ \varphi=0 & \text { in } B_{R} \times\{T\}\end{cases}
$$

for any $R>R_{0}$.

Observe that, by the maximum principle, $\varphi$ is bounded in $Q_{R, T}$ uniformly with respect to $R$. We put

$$
M:=\max _{\bar{Q}_{R, T}}|\varphi|
$$

and consider the problem

$$
\begin{cases}\rho \partial_{t} \chi+\Delta \chi=0 & \text { in }\left(B_{R} \backslash \bar{B}_{R_{0}}\right) \times(0, T)=: K_{R, T}, \\ \chi=M & \text { in } \partial B_{R_{0}} \times(0, T), \\ \chi=0 & \text { in } \partial B_{R} \times(0, T), \\ \chi=z & \text { in }\left(B_{R} \backslash \bar{B}_{R_{0}}\right) \times\{T\},\end{cases}
$$

where

$$
z(x):=M \frac{|x|^{2-n}-R^{2-n}}{R_{0}^{2-n}-R^{2-n}} \quad\left(x \in B_{R} \backslash \bar{B}_{R_{0}}\right) .
$$

It is easily seen that $\varphi$ is a subsolution of (2.27) (recall that $\operatorname{supp} F \subseteq B_{R_{0}} \times(0, T)$, so that $\partial_{t} F \equiv 0$ in $\left.K_{R, T}\right)$. On the other hand, the function $z$ defined in (2.28) is a solution of the same problem; hence, by the maximum principle, $\varphi \leq z$ in $K_{R, T}$. Since $\varphi=z$ in $\partial B_{R} \times(0, T)$, we also have

$$
\partial_{\nu} \varphi>-\frac{(n-2) M}{R_{0}^{2-n}-R^{2-n}} R^{1-n} \quad \text { in } \partial B_{R} \times(0, T) .
$$

Similarly, $\varphi \geq-z$ in $K_{R, T}$, and

$$
\partial_{\nu} \varphi<\frac{(n-2) M}{R_{0}^{2-n}-R^{2-n}} R^{1-n} \quad \text { in } \partial B_{R} \times(0, T) .
$$

Using (2.29) and (2.30), from (2.26) we deduce the inequality

$$
\begin{aligned}
\left|\int_{0}^{T} \int_{\partial B_{R}} w \partial_{\nu} \psi d \sigma d t\right| & \leq \frac{(n-2) M}{R_{0}^{2-n}-R^{2-n}} R^{1-n} \int_{0}^{T} \int_{\partial B_{R}}|W| d \sigma d t \\
& =C_{R} \int_{0}^{T} f_{\partial B_{R}}|W| d \sigma d t
\end{aligned}
$$

with

$$
C_{R}:=\frac{(n-2) M\left|\partial B_{1}\right|}{R_{0}^{2-n}-R^{2-n}} .
$$


Since, as $R \rightarrow \infty$,

$$
f_{\partial B_{R}}|W(x, t)| d \sigma \rightarrow 0
$$

uniformly with respect to $t \in[0, T]$ (see $(2.22)$ ), the above inequality shows that $(2.25)$ is true. This completes the proof.

Remark 2.2. The above uniqueness result implies that the entire family $\left\{u_{R}\right\}$ of solutions of problem (2.9) converges as $R \rightarrow \infty$ to a bounded solution of (1.1) satisfying (1.5).

\section{§3. Neumann conditions: Proofs}

First, we prove Theorem 1.4.

Proof of Theorem 1.4. Let $T>0$ be fixed arbitrarily. For any $R>0$, we set $u_{0, R}:=$ $\zeta_{R} u_{0}$, where $\zeta_{R} \in C_{0}^{\infty}\left(B_{R}\right), 0 \leq \zeta_{R} \leq 1, \zeta_{R}=1$ in $B_{R / 2}$. Consider the auxiliary problem

$$
\begin{cases}\rho \partial_{t} u=\Delta u & \text { in } Q_{R, T} \\ \partial_{\nu} u=0 & \text { in } \partial B_{R} \times(0, T) \\ u=u_{0, R} & \text { in } B_{R} \times\{0\}\end{cases}
$$

The existence and uniqueness of solutions, as well as comparison results for problem (3.1), are proved by the usual methods (see, e.g., [12]).

Let $u_{R}$ denote a unique solution of problem (3.1); by comparison, we have

$$
\left|u_{R}\right| \leq\left\|u_{0}\right\|_{\infty} \quad \text { in } Q_{R, T} .
$$

Let $R \rightarrow \infty$. By compactness, there exists a sequence $\left\{u_{R_{k}}\right\}$ that converges uniformly on any compact subset of $\mathbb{R}^{n} \times(0, T)$ to a bounded solution $\hat{u}$ of problem (1.1); in fact, we have

$$
|\hat{u}| \leq\left\|u_{0}\right\|_{\infty} \quad \text { in } \mathbb{R}^{n} \times(0, T)
$$

for any $T>0$. Moreover, (3.1) implies that

$$
\int_{B_{R_{k}}} \rho u_{R_{k}}(x, t) d x=\int_{B_{R_{k}}} \rho u_{0, R_{k}}(x) d x
$$

for any $R_{k}$. Since $\rho \in L^{1}\left(\mathbb{R}^{n}\right)$ by assumption (see $\left(\mathrm{H}_{2}\right)$ ), inequality (3.2) allows us to pass to the limit as $R_{k} \rightarrow \infty$ in the above identity; thus, $\hat{u} \in \mathcal{N}$.

The remaining claims concerning $\hat{u}$ follow easily from the energy estimate:

$$
\frac{1}{2} \int_{B_{R_{k}}} \rho u_{R_{k}}^{2}(x, t) d x+\int_{0}^{t} \int_{B_{R_{k}}}\left|\nabla u_{R_{k}}(x, \tau)\right|^{2} d x d \tau=\frac{1}{2} \int_{B_{R_{k}}} \rho u_{0, R_{k}}^{2} d x \quad(t>0)
$$

as $R_{k} \rightarrow \infty$. This completes the proof.

Proof of Theorem 1.5. Let $u_{1}, u_{2}$ be two bounded solutions of class $\mathcal{N}$ of problem (1.1), and let $w:=u_{1}-u_{2}$. Observe that

$$
\int_{\mathbb{R}^{n}} \rho w(x, t) d x=0 \quad \text { for any } t \geq 0
$$

Set

$$
W(x, t):=\int_{0}^{t} w(x, \tau) d \tau \quad((x, t) \in S) .
$$

By Theorem 1.1, there exists $A \in \operatorname{Lip}\left(\overline{\mathbb{R}}_{+}\right)$such that

$$
\lim _{R \rightarrow \infty} f_{\partial B_{R}}|W(x, t)-A(t)| d \sigma=0
$$


uniformly with respect to $t \in[0, T]$. Let $a \equiv A^{\prime} \in L^{\infty}\left(\mathbb{R}_{+}\right)$, and let $a_{R}$ denote a smooth approximation of $a$ such that $a_{R}(0)=0$ and $\left\|a_{R}\right\|_{\infty} \leq\|a\|_{\infty}$ for any $R>0$, and $a_{R} \rightarrow a$ in $L^{1}(0, T)$ for any $T>0$ as $R \rightarrow \infty$. For any fixed $T>0$, we consider the problem

$$
\begin{cases}\rho \partial_{t} w=\Delta w & \text { in } Q_{R, T} \\ w=a_{R}(\cdot) & \text { in } \partial B_{R} \times(0, T), \\ w=0 & \text { in } B_{R} \times\{0\}\end{cases}
$$

and let $w_{R}$ be its solution. Arguing as in the proof of Theorem 1.2, and using Remark 2.2 , we see that

$$
w=\lim _{R \rightarrow \infty} w_{R}
$$

uniformly on compact subsets of $\mathbb{R}^{n} \times(0, T)$.

Now we observe that any solution $w_{R}$ of problem (3.4) satisfies the identity

$$
\int_{0}^{t} \int_{B_{R}}\left\{\rho w_{R} \partial_{t} \phi-\nabla w_{R} \nabla \phi\right\} d x d \tau=\int_{B_{R}} \rho w_{R}(x, t) \phi(x, t) d x
$$

for any $t \in(0, T]$ and any $\phi \in \operatorname{Lip}\left([0, T] ; C^{1}\left(\bar{B}_{R}\right)\right)$ such that $\phi=0$ on $\partial B_{R} \times[0, T]$. As in [13], we define

$$
\varphi_{R}(x, t):=-\int_{t}^{T} w_{R}(x, \tau) d \tau+\int_{t}^{T} a_{R}(\tau) d \tau \quad\left((x, t) \in \bar{Q}_{R, T}\right) .
$$

It is easily seen that

- $\varphi_{R}=0$ in $\left(B_{R} \times\{T\}\right) \cup\left(\partial B_{R} \times(0, T)\right)$;

- we have

$$
\begin{aligned}
& \nabla \varphi_{R}(x, t)=-\int_{t}^{T} \nabla w_{R}(x, \tau) d \tau \\
& \partial_{t} \varphi_{R}(x, t)=w_{R}(x, t)-a_{R}(t) \quad \text { in } Q_{R, T},
\end{aligned}
$$

whence $\varphi_{R} \in \operatorname{Lip}\left([0, T] ; C^{1}\left(\bar{B}_{R}\right)\right)$. We set $t=T$ and $\phi=\varphi_{R}$ in (3.6); using the above properties of $\varphi_{R}$, we obtain

$$
\begin{aligned}
\int_{0}^{T} \int_{B_{R}} \rho w_{R}^{2}(x, t) d x d t & +\int_{0}^{T} \int_{B_{R}} \nabla w_{R}(x, t)\left\{\int_{t}^{T} \nabla w_{R}(x, \tau) d \tau\right\} d x d t \\
& =\int_{0}^{T} a_{R}(t) \int_{B_{R}} \rho w_{R}(x, t) d x d t
\end{aligned}
$$

Since

$$
\nabla w_{R}(x, t)\left\{\int_{t}^{T} \nabla w_{R}(x, \tau) d \tau\right\}=-\frac{1}{2} \partial_{t}\left|\int_{t}^{T} \nabla w_{R}(x, \tau) d \tau\right|^{2}
$$

(3.7) implies

$$
\begin{aligned}
\int_{0}^{T} \int_{B_{R}} \rho w_{R}^{2}(x, t) d x d t & +\frac{1}{2} \int_{B_{R}}\left|\int_{0}^{T} \nabla w_{R}(x, t) d t\right|^{2} d x \\
& =\int_{0}^{T} a_{R}(t) \int_{B_{R}} \rho w_{R}(x, t) d x d t
\end{aligned}
$$

Thus

$$
\int_{0}^{T} \int_{B_{R}} \rho w_{R}^{2}(x, t) d x d t \leq \int_{0}^{T} a_{R}(t) \int_{B_{R}} \rho w_{R}(x, t) d x d t
$$


for any $R>0$. Since

$$
\lim _{R \rightarrow \infty} \int_{B_{R}} \rho w_{R}(x, t) d x=\int_{\mathbb{R}^{n}} \rho w(x, t) d x=0
$$

for any $t \in[0, T]$, inequality (3.9) allows us to conclude that

$$
\lim _{R \rightarrow \infty} \int_{0}^{T} \int_{B_{R}} \rho w_{R}^{2}(x, t) d x d t=\int_{0}^{T} \int_{\mathbb{R}^{n}} \rho w^{2}(x, t) d x d t=0 .
$$

Consequently, $w \equiv 0$, so that $u_{1}=u_{2}$ in $\mathbb{R}^{n} \times(0, T)$ for any $T>0$; the result follows.

Remark 3.1. It is worth pointing out an alternative proof of Theorem 1.5. Let $w, W, w_{R}$ be defined as in the proof above, and let

$$
W_{R}(x, t):=\int_{0}^{t} w_{R}(x, \tau) d \tau \quad\left((x, t) \in Q_{R, T}\right) .
$$

Observe that, by relation (3.5) (which is fulfilled uniformly on compact subsets), $W_{R} \rightarrow$ $W$ in $\mathbb{R}^{n} \times[0, T]$ as $R \rightarrow \infty$.

Obviously, (3.4) implies that $W_{R}$ satisfies the problem

$$
\begin{cases}\rho \partial_{t} W=\Delta W & \text { in } Q_{R, T}, \\ W=A_{R}(\cdot) & \text { in } \partial B_{R} \times(0, T), \\ W=0 & \text { in } B_{R} \times\{0\}\end{cases}
$$

for any $R>0$, with $A_{R}$ defined as in (2.12). Multiplying the first equation in (3.11) by $W_{R}-A_{R}(\cdot)$ and integrating in time, we easily get

$$
\begin{aligned}
\frac{1}{2} \int_{B_{R}} \rho W_{R}^{2}(x, t) d x & +\int_{0}^{t} \int_{B_{R}}\left|\nabla W_{R}(x, \tau)\right|^{2} d x d \tau \\
& =\int_{0}^{t} A_{R}(\tau) \int_{B_{R}} \rho w_{R}(x, \tau) d x d \tau \quad(t \in[0, T]),
\end{aligned}
$$

whence

$$
\frac{1}{2} \int_{B_{R}} \rho W_{R}^{2}(x, t) d x \leq \int_{0}^{t} A_{R}(\tau) \int_{B_{R}} \rho w_{R}(x, \tau) d x d \tau
$$

for any $R>0, t \in[0, T]$. Using (3.10) and (3.13), we obtain

$$
\lim _{R \rightarrow \infty} \int_{B_{R}} \rho W_{R}^{2}(x, t) d x=\int_{\mathbb{R}^{n}} \rho W^{2}(x, t) d x=0
$$

for any $t \in[0, T]$. Again, this shows that $w \equiv 0$ in $\mathbb{R}^{n} \times(0, T)$ for any $T>0$, and the conclusion follows.

To prove Theorem 1.7, we need the following statement.

Lemma 3.2. Let $u$ be any bounded solution of class $\mathcal{N}$ of problem (1.1). Then

$$
\lim _{t \rightarrow \infty} \int_{B_{R}}|\nabla u(x, t)|^{2} d x=0
$$

for any $R>0$.

The proof of Lemma 3.2 is a standard adaptation of the proof given in [6] for $n=1,2$; we omit this.

In what follows, we set

$$
f_{B_{R}} v d x:=\frac{1}{\left|B_{R}\right|} \int_{B_{R}} v d x
$$


Proof of Theorem 1.7. Fixing $R>0$, we define

$$
D(x, t):=u(x, t)-f_{B_{R}} u(x, t) d x \quad\left((x, t) \in \mathbb{R}^{n} \times[0, \infty)\right) .
$$

The Poincaré inequality and Lemma 3.2 yield

$$
\lim _{t \rightarrow \infty} \int_{B_{R}}|D(x, t)|^{2} d x \leq C_{R}^{\prime} \lim _{t \rightarrow \infty} \int_{B_{R}}|\nabla u(x, t)|^{2} d x=0
$$

with some constant $C_{R}^{\prime}>0$.

By classical results, on the compact subsets of $\mathbb{R}^{n}$ the solution $u(\cdot, t)$ satisfies Hölder estimates uniformly with respect to time in $[\tau, \infty)(\tau>0)$. Then, by the Ascoli-Arzelà theorem, there exists a sequence $\left\{t_{k}\right\}, t_{k} \rightarrow \infty$, and a function $\tilde{u} \in C\left(\mathbb{R}^{n}\right)$ such that

$$
\lim _{t_{k} \rightarrow \infty} u\left(\cdot, t_{k}\right)=\tilde{u}
$$

uniformly on the compact subsets of $\mathbb{R}^{n}$. It follows that

$$
\lim _{t_{k} \rightarrow \infty} f_{B_{R}} u\left(x, t_{k}\right) d x=f_{B_{R}} \tilde{u} d x .
$$

From (3.15)-(3.17) we deduce that

$$
\left\|\tilde{u}-f_{B_{R}} \tilde{u} d x\right\|_{L^{2}\left(B_{R}\right)}=\lim _{t_{k} \rightarrow \infty}\left\|D\left(\cdot, t_{k}\right)\right\|_{L^{2}\left(B_{R}\right)}=0
$$

whence $\tilde{u}=$ constant. Using the conservation law (1.6), we conclude that

$$
\lim _{t_{k} \rightarrow \infty} u\left(\cdot, t_{k}\right)=\frac{\int_{\mathbb{R}^{n}} \rho u_{0} d x}{\int_{\mathbb{R}^{n}} \rho d x} .
$$

It is easily seen that the same limit is attained along any diverging sequence $\left\{t_{k}\right\}$; the conclusion follows.

Acknowledgements. We should like to thank Professor N. N. Ural'tseva for helpful discussions, and Professor S. S. Grigoryan, Institute of Mechanics, Moscow State University, for pointing out to us the physical interest of boundary conditions of Neumann type at infinity.

The first author thanks the Department of Mathematics "G. Castelnuovo" of the University of Rome "La Sapienza" for warm hospitality and partial support.

\section{REFERENCES}

[1] H. Brezis and S. Kamin, Sublinear elliptic equations in $\mathbb{R}^{n}$, Manuscripta Math. 74 (1992), 87-106. MR1141779 (93f:35062)

[2] S. D. Eidelman and S. Kamin, On stabilization of solutions of the Cauchy problem for parabolic equations degenerating at infinity, Asymptot. Anal. 45 (2005), 55-71. MR2181259 (2006i:35124)

[3] S. D. Eidelman, S. Kamin, and F. Porper, Uniqueness of solutions of the Cauchy problem for parabolic equations degenerating at infinity, Asymptot. Anal. 22 (2000), 349-358. MR1753769 (2001c:35094)

[4] D. Eidus, The Cauchy problem for the nonlinear filtration equation in an inhomogeneous medium, J. Differential Equations 84 (1990), 309-318. MR1047572 (91d:35172)

[5] D. Eidus and S. Kamin, The filtration equation in a class of functions decreasing at infinity, Proc. Amer. Math. Soc. 120 (1994), 825-830. MR1169025 (94e:35070)

[6] M. Guedda, D. Hilhorst, and M. A. Peletier, Disappearing interfaces in nonlinear diffusion, Adv. Math. Sci. Appl. 7 (1997), 695-710. MR1476273 (98j:35094)

[7] K. Ishige and M. Murata, An intrinsic metric approach to uniqueness of the positive Cauchy problem for parabolic equations, Math. Z. 227 (1998), 313-335. MR1609065 (99c:35088)

[8] S. Kamin and P. Rosenau, Nonlinear diffusion in finite mass medium, Comm. Pure Appl. Math. 35 (1982), 113-127. MR0637497 (83m:35079) 
[9] S. Kamin, R. Kersner, and A. Tesei, On the Cauchy problem for a class of parabolic equations with variable density, Atti Accad. Naz. Lincei Cl. Sci. Fis. Mat. Natur. Rend. Lincei (9) Mat. Appl. 9 (1998), 279-298. MR1722787 (2000j:35125)

[10] R. Kersner and A. Tesei, Well-posedness of initial value problems for singular parabolic equations, J. Differential Equations 199 (2004), 47-76. MR2041511 (2005d:35121)

[11] M. Murata, Nonuniqueness of the positive Dirichlet problem for parabolic equations in cylinders, J. Funct. Anal. 135 (1996), 456-487. MR1370610 (97a:35085)

[12] O. A. Ladyzhenskaya, V. A. Solonnikov, and N. N. Ural'tseva, Linear and quasilinear equations of parabolic type, "Nauka", Moscow, 1967; English transl., Transl. Math. Monogr., vol. 23, Amer. Math. Soc., Providence, RI, 1968. MR0241822 (39:3159b)

[13] O. A. Oleĭnik, A. S. Kalashnikov, and Y.-L. Chzhou, The Cauchy problem and boundary problems for equations of the type of non-stationary filtration, Izv. Akad. Nauk SSSR Ser. Mat. 22 (1958), no. 5, 667-704. (Russian) MR0099834 (20:6271)

[14] Y. Pinchover, On uniqueness and nonuniqueness of the positive Cauchy problem for parabolic equations with unbounded coefficients, Math. Z. 223 (1996), 569-586. MR1421956 (97j:35059)

[15] M. A. Pozio and A. Tesei, On the uniqueness of bounded solutions to singular parabolic problems, Discrete Contin. Dyn. Syst. 13 (2005), 117-137. MR2128795 (2006a:35178)

[16] G. Reyes and J. L. Vazquez, A weighted symmetrization for nonlinear elliptic and parabolic equations in inhomogeneous media, Preprint, 2005. MR2250171 (2007e:35007)

[17] A. Tesei, On uniqueness of the positive Cauchy problem for a class of parabolic equations, Current Problems of Analysis and Mathematical Physics (Taormina, 1998), Aracne, Rome, 2000, pp. 145160 (Italian). MR1809023 (2001m:35167)

School of Mathematical Sciences, Tel-Aviv University, Ramat-Aviv, 69978, Tel-Aviv, IsRAEL

E-mail address: kamin@post.tau.ac.il

Dipartimento di Matematica "G. Castelnuovo", Università di Roma "La Sapienza", P. le

A. Moro 5, I-00185 Roma, Italia

E-mail address: pozio@mat.uniroma1.it

Dipartimento di Matematica "G. Castelnuovo", Università di Roma "La Sapienza", P. le

A. Moro 5, I-00185 Roma, Italia

E-mail address: tesei@mat.uniroma1.it

Received 1/DEC/2005

Originally published in English 